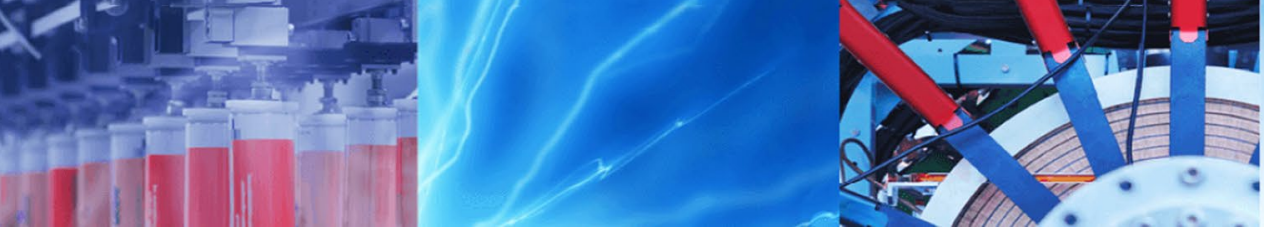

Research Article

\title{
Permanent magnet synchronous motor: chaos control using single controller, synchronization and circuit implementation
}

\author{
André Cheukem ${ }^{1}$ - Alex Stephane Kemnang Tsafack ${ }^{2}$ S Sifeu Takougang Kingni ${ }^{3}$. Chéagé Chamgoué André ${ }^{4}$. \\ Justin Roger Mboupda Pone ${ }^{1}$
}

Received: 15 October 2019 / Accepted: 6 February 2020 / Published online: 15 February 2020

(c) Springer Nature Switzerland AG 2020

\begin{abstract}
The aim of this paper is to address the problem of the electronic implementation of chaos control using a single controller and synchronization of chaotic permanent magnet synchronous motor (PMSM). Firstly, different dynamical behaviors of the PMSM including steady state, periodic and chaotic behaviors are found using numerical methods such as twodimensional largest Lyapunov exponents graph associated with two parameters of PMSM. Secondly, two simple and single controllers are designed and added to the chaotic PMSM in order to suppress chaotic behavior. The performance of the two proposed simple and single controllers is illustrated by numerical simulations. Thirdly, controllers are designed to achieve synchronization of unidirectional coupled identical chaotic PMSMs. Numerical simulations are also used to verify the effectiveness of the synchronization. Finally, the existence of chaos in PMSM and the physical feasibility of the proposed two simple and single controllers as well as the chaos synchronization are validated through the circuit implementation on OrCAD-PSpice software. The circuit implementation results comply fairly with those of the numerical simulation results and establish that the existence of chaotic behavior in the PMSM and the achievement of chaos synchronization in unidirectional coupled identical PMSMs designed and the two single controllers designed are effective and successful in suppressing chaotic behavior in PMSM.
\end{abstract}

Keywords Permanent magnet synchronous motor · Chaos · Chaos control · Single controller · Synchronization · Circuit implementation

\section{Introduction}

A PMSM is a kind of high-efficient and high-powered motor which is widely used in motor drive, various servo systems and household appliances $[4,9,11,15,24,28$, $36]$, due to its high torque per volume ratio and efficiency along with low maintenance $[26,39]$. Unfortunately, it has been proven recently that the dynamics performance of PMSM is sensitive to system parameter perturbations in the whole operation process $[29,31]$. The chaotic attractors in PMSM were reported for the first time in Refs. [16-18]. Lyapunov spectrum and dynamical analysis of a PMSM in the presence of virtual control force were reported in Ref. [34]. Multistability and hidden attractors were found in PMSM Ref. [30]. Chaotic behavior of the PMSM is undesirable since it can destroy the stabilization of the motor and even induce the drive system to collapse Ref. [33]. Consequently, chaos control with the aim of suppressing the

André Cheukem, acheukem05@gmail.com | ${ }^{1}$ Research Unit of Automation and Applied Computer, Department of Electrical Engineering, IUT-FV, University of Dschang, P.O. Box: 134, Bandjoun, Cameroon. ${ }^{2}$ Research Unit of Condensed Matter of Electronics and Signal Processing, Department of Physics, Faculty of Sciences, University of Dschang, P.O. Box: 67, Dschang, Cameroon. ${ }^{3}$ Department of Mechanical, Petroleum and Gas Engineering, Faculty of Mines and Petroleum Industries, University of Maroua, P.O. Box: 46, Maroua, Cameroon. ${ }^{4}$ Department of Basic science, School of Geology and Mining Engineering, University of Ngaoundere, P.O. Box: 115, Meiganga, Cameroon. 
undesired chaotic behavior of PMSM has attracted increasing attention in order to further improve the performance of the system in the industrial and academic community $[6,22,35]$. Chaos control in PMSM has been implemented with several methods. Feedback control method [2, 12, $14]$, adaptive fuzzy control $[5,21,37,38]$, simple sliding mode adaptive control $[7,23]$, adaptive neural sliding mode control [33], optimal Lyapunov exponents' placement [1], passive control [25], impulsive control [6, 10] and finite-time stability theory [27] were used for the control of chaotic behavior in PMSM. However, the methods mentioned above have some shortcomings. For example, the control objective is required to be an equilibrium point in feedback control methods and sliding mode control methods can be easily influenced by chattering. Moreover, the fuzzy rules applied in control design are over-dependent on experiences.

Most of the existing techniques to suppress chaotic behavior in PMSM use nonlinear and complicated controls. Studies of simple control laws based on single-state feedback remain scarce [13]. The single-state feedback control method is very simple, extremely concise and easy to implement. Therefore, the main contribution of this paper

\section{Rate equations of permanent magnet synchronous motor}

The mathematical model of the PMSM which is based on the $d$ (direct)-q (quadrature) axis model is summarized in Refs. $[17,18]$ as follows:

$\frac{\mathrm{d} \tilde{i}_{d}}{\mathrm{~d} \tilde{t}}=\left(\tilde{u}_{\mathrm{d}}-R_{1} \tilde{i}_{\mathrm{d}}+\tilde{\omega} L_{\mathrm{q}} \tilde{i}_{\mathrm{d}}\right) / L_{\mathrm{d}}$

$\frac{\mathrm{d} \tilde{i}_{\mathrm{q}}}{\mathrm{d} \tilde{t}}=\left(\tilde{u}_{\mathrm{q}}-R_{1} \tilde{i}_{\mathrm{q}}-\tilde{\omega} L_{\mathrm{d}} \tilde{i}_{\mathrm{d}}\right) / L_{\mathrm{q}}$

$\frac{\mathrm{d} \tilde{\omega}}{\mathrm{d} \tilde{t}}=\left[\eta_{\mathrm{p}} \psi_{\mathrm{r}} \tilde{i}_{\mathrm{q}}+\eta_{\mathrm{q}}\left(L_{\mathrm{d}}-L_{\mathrm{q}}\right) \tilde{i}_{\mathrm{d}} \tilde{i}_{\mathrm{q}}-\tilde{T}_{\mathrm{L}}-\beta \tilde{\omega}\right] / J$,

where $\tilde{i}_{\mathrm{d}}, \tilde{i}_{\mathrm{q}}$ are the $\mathrm{d}$ - and q-axis rotor currents, $\tilde{\omega}$ is the mechanical rotor speed, $\tilde{u}_{d}, \tilde{u}_{q}$ are the stator voltage on $\mathrm{d}$-q-axis, $\tilde{T}_{\mathrm{L}}$ is the mechanical load torque, $L_{\mathrm{d}}, L_{\mathrm{q}}$ are $\mathrm{d}$ - and $\mathrm{q}$-axis stator inductors, $\psi_{\mathrm{r}}$ is the permanent magnet flux, $R_{1}$ is the stator winding resistance, $J$ is the polar moment of inertia, $\beta$ is the viscous damping coefficient and $\eta_{\mathrm{p}}$ is the number of pole pairs. By defining a set of new variables as:

$\tau=L_{\mathrm{q}} / R_{1}, \tilde{t}=\tau t, k=\beta /\left(\tau \eta_{\mathrm{p}} \psi_{\mathrm{r}}\right), \tilde{u}_{\mathrm{d}}=u_{\mathrm{d}} /\left(k R_{1}\right), \tilde{u}_{\mathrm{q}}=u_{\mathrm{q}} /\left(k R_{1}\right), \gamma=\psi_{\mathrm{r}} /\left(k L_{\mathrm{q}}\right), \sigma=\beta \tau / J$,

$\tilde{T}_{\mathrm{L}}=T_{\mathrm{L}} \tau^{2} / J, b=L_{\mathrm{q}} / L_{\mathrm{d}}, \delta=\eta_{\mathrm{p}} b \tau^{2} k^{2}\left(L_{\mathrm{d}}-L_{\mathrm{q}}\right) / J, x=\tilde{i}_{\mathrm{d}}, y=\tilde{i}_{\mathrm{q}}, z=\tilde{\omega}$,

is to develop and study single, simple and effective controllers based on the principle of Lyapunov's method for asymptotic global stability. In addition, authors investigate the chaos synchronization in unidirectional coupled identical PMSMs because chaos synchronization has a great number of applications in many areas such as physics [19], biology [32], signal engineering [8] and ecological science [3].

In this paper, chaos control via single simple and state feedback control and synchronization of chaotic PMSM are analytically, numerically and electronically analyzed. In Sect. 2, the chaotic PMSM is described. In Sect. 3, two single-state feedback controllers are employed for achieving the control of the chaotic PMSM. In Sect. 4, the feedback controller method is used for achieving synchronization in unidirectional coupled identical chaotic PMSMs. In Sect. 5, electronic circuits are implemented to check the existence of chaotic behavior in PMSM and the effectiveness of the single-state feedback control method and the synchronization in unidirectional coupled identical chaotic PMSMs. Finally, conclusions are given in Sect. 6 . the dimensionless form of system (1) can be rewritten as follows:

$\frac{\mathrm{d} x}{\mathrm{~d} t}=u_{\mathrm{d}}-x+y z$

$\frac{\mathrm{d} y}{\mathrm{~d} t}=u_{\mathrm{q}}-y-x z+\gamma z$

$\frac{\mathrm{d} z}{\mathrm{~d} t}=\sigma(y-z)+\delta x y-T_{\mathrm{L}}$

Considering the special case where there are no loads $\left(u_{\mathrm{d}}=u_{\mathrm{q}}=T_{\mathrm{L}}=0\right)$ and the PMSM is a smooth air gap $\left(L_{d}=L_{q}\right)$, the system (3) becomes

$\frac{\mathrm{d} x}{\mathrm{~d} t}=-x+y z$

$\frac{\mathrm{d} y}{\mathrm{~d} t}=-y-x z+\gamma z$

$\frac{d z}{d t}=\sigma(y-z)$. 
The system (4) is invariant under the transformation: $(x, y, z) \Leftrightarrow(x,-y,-z)$, and it is dissipative if $\nabla V=\frac{\partial(\mathrm{d} x / \mathrm{d} t)}{\partial x}+\frac{\partial(\mathrm{d} y / \mathrm{d} t)}{\partial y}+\frac{\partial(\mathrm{d} z / \mathrm{d} t)}{\partial z}=-(\sigma+2)<0$. It has only one equilibrium point $O(0,0,0)$ if $\gamma \leq 1$ and three e quilibrium points $O(0,0,0)$, $E_{1,2}(\gamma-1, \pm \sqrt{\gamma-1}, \pm \sqrt{\gamma-1})$ [18]. The linear stability analysis of the system (4) revealed that the equilibrium points displayed Pitchfork and Hopf bifurcations [18]. When the parameters $\sigma, \gamma$ are varied, the PMSM can be expected to exhibit steady state, periodic and chaotic behaviors. In order to identify the dynamical behaviors in PMSM, a two-dimensional largest Lyapunov exponent (LLE) diagram is depicted in Fig. 1. Such a diagram is useful when PMSM is examined experimentally. It can serve as a guide for appropriate operating parameters to target a particular dynamical behavior.

In Fig. 1, colors are associated with the magnitude of the LLE as follows: Negative LLE is indicated by a blue scale, and positive LLE is indicated by a continuously changing between light blue, green, yellow and red scales. On LLE diagram in $(\gamma, \sigma)$ space, periodic and steady states regions are characterized by blue color and chaotic regions are characterized by light blue, green, yellow and red colors. In Refs. [18, 30], the authors showed that PMSM described by system (4) exhibited a period-doubling bifurcation to chaotic behavior. For $\sigma=5.46$ and $\gamma=20$, system (4) can display a double-scroll chaotic attractor as shown in Fig. 2.

\section{Chaos control of permanent magnet synchronous motor using a single controller}

A single-state feedback controller is used in this section to stabilize the chaotic behavior found in PMSM. Therefore, two single controllers are mathematically designed by

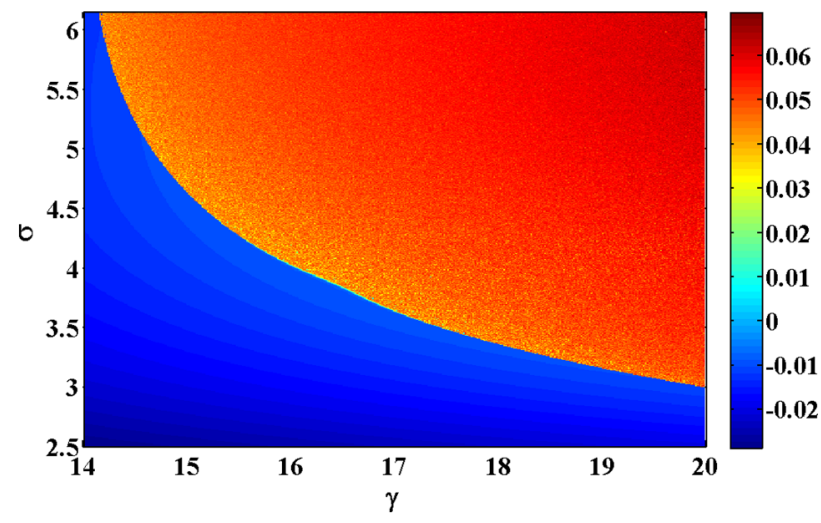

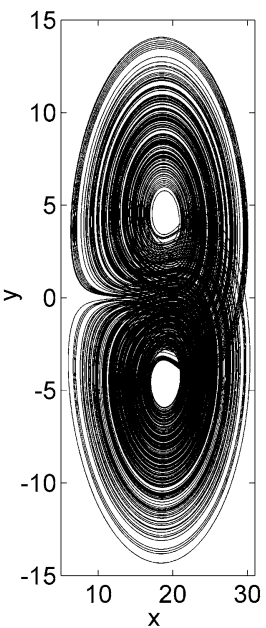
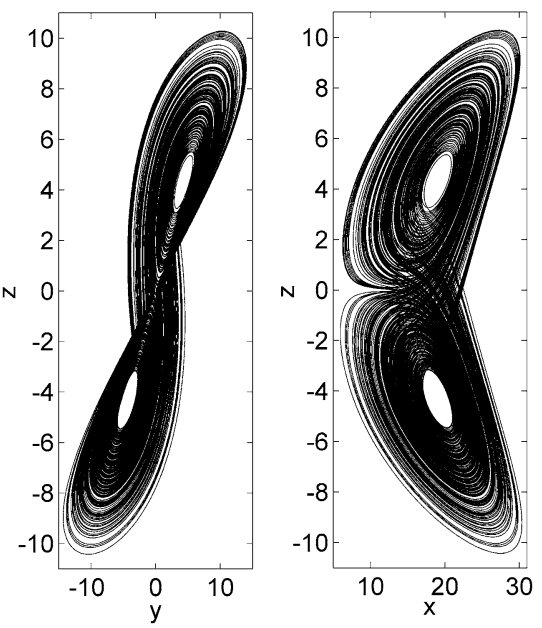

Fig. 2 Two-dimensional phase portrait of system (4) for $\sigma=5.46$ and $\gamma=20$. The initial conditions are $(x(0), y(0), z(0))=(0.01,0.01$, $0.01)$

using the principle of Lyapunov's method for asymptotic global stability [20].

\subsection{Proposed controller 1}

In this subsection, the controller $u_{1}$ is added to Eq. (4b):

$\frac{\mathrm{d} x}{\mathrm{~d} t}=-x+y z$

$\frac{\mathrm{d} y}{\mathrm{~d} t}=-y-x z+\gamma z+u_{1}$

$\frac{\mathrm{d} z}{\mathrm{~d} t}=\sigma(y-z)$,

where $u_{1}=z(x-\gamma)$. Substituting the expression of the controller $u_{1}$ into the controlled system (5) becomes

$\frac{\mathrm{d} x}{\mathrm{~d} t}=-x+y z$

$\frac{d y}{d t}=-y$,

$\frac{d z}{d t}=\sigma(y-z)$

The solution of Eq. (6b) is $y(t)=y(0) e^{-t}$. That yields $\lim _{t \rightarrow \infty} y(t)=0$. Thus, system (6) can be reduced as follows:

$\frac{\mathrm{d} x}{\mathrm{~d} t}=-x$,

Fig. 1 (Color online) Two-parameter LLE diagram in $(\gamma, \sigma)$ space 
$\frac{d z}{d t}=-\sigma z$

The solution of system (7) can be rewritten as follows:

$x(t)=x(0) e^{-t}$,

$z(t)=z(0) e^{-\sigma t}$

That yields $\lim _{t \rightarrow \infty} x(t)=0$ and $\lim _{t \rightarrow \infty} z(t)=0$. Therefore, the following theorem is given:

Theorem 1 The chaotic behavior found in PMSM can be controlled using the controller $u_{1}=z(x-\gamma)$.

Proof The proof is obvious, so leave it out.

The time series of the state responses and the output of the controller are shown in Fig. 3.

From Fig. 3 , the controller $u_{1}$ is activated at $t \geq 1400$. It is noted that Theorem 1 is effective.

\subsection{Proposed controller 2}

In this subsection, the controller $u_{2}$ is added to Eq. (4c):

$\frac{\mathrm{d} x}{\mathrm{~d} t}=-x+y z$ $\frac{\mathrm{d} y}{\mathrm{~d} t}=-y-x z+\gamma z$

$\frac{\mathrm{d} z}{\mathrm{~d} t}=\sigma(y-z)+u_{2}$

where $u_{2}=-\sigma y$. Substituting the expression of the controller $u_{2}$ into the controlled system (9) becomes

$\frac{\mathrm{d} x}{\mathrm{~d} t}=-x+y z$

$\frac{\mathrm{d} y}{\mathrm{~d} t}=-y-x z+\gamma z$

$\frac{\mathrm{d} z}{\mathrm{~d} t}=-\sigma z$

The solution of Eq. (10c) is $z(t)=z(0) e^{-\sigma t}$. That yields $\lim _{t \rightarrow \infty} z(t)=0$. Thus, the system (10) can be reduced as follows:

$\frac{\mathrm{d} x}{\mathrm{~d} t}=-x$

$\frac{\mathrm{d} y}{\mathrm{~d} t}=-y$.
Fig. 3 Time series of $x, y, z$ and the output of the controller $u_{1}$ for $\sigma=5.46$ and $\gamma=20$. The initial conditions are $(x(0)$, $y(0), z(0))=(0.01,0.01,0.01)$
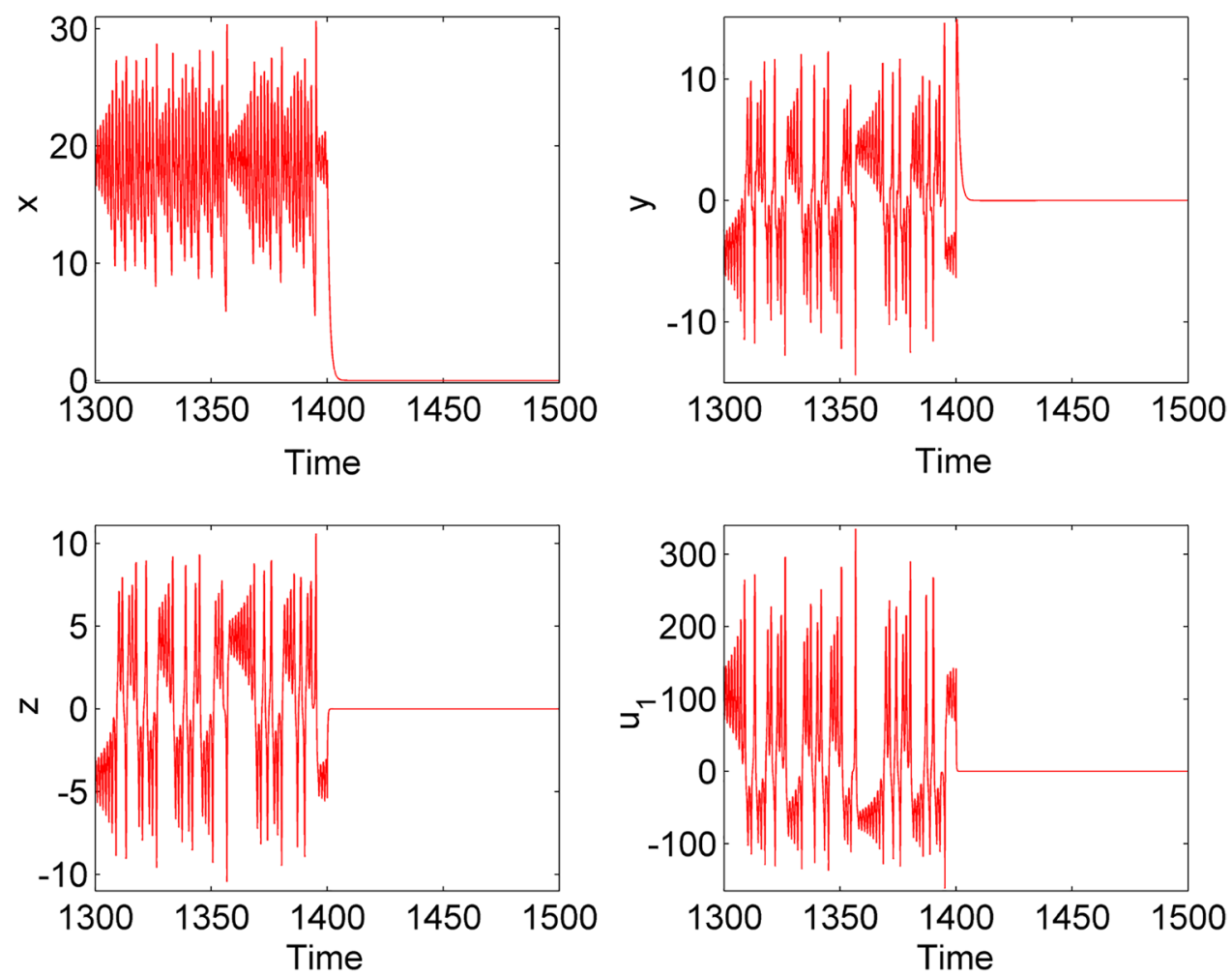
The solution of system (11) can be rewritten as follows:

$x(t)=x(0) e^{-t}$,

$y(t)=y(0) e^{-t}$.

That yields $\lim _{t \rightarrow \infty} x(t)=0$ and $\lim _{t \rightarrow \infty} y(t)=0$. Therefore, the following theorem is given:

Theorem 2 The chaotic behavior found in PMSM can be controlled using the controller $u_{2}=-\sigma y$.

Proof The proof is obvious, so leave it out.

The time series of the state responses and the output of the controller are shown in Fig. 4.

From Fig. 4 , the controller $u_{2}$ is activated at $t \geq 1400$. It is noted that Theorem 2 is effective. It is interesting to point out here that from a practical realization point of view, the single controller 2 is preferred because of the inclusion of one state variable (i.e., y) signifying a lesser requirement of sensing devices during their fabrication. Hence, this makes the system become cheap.

\section{Chaos synchronization of unidirectional coupled identical permanent magnet synchronous motors}

In this section, a feedback control method is used to synchronize unidirectional coupled identical chaotic PMSMs. The drive and the response PMSMs are expressed, respectively, as follows:

$\frac{\mathrm{d} x_{1}}{\mathrm{~d} t}=-x_{1}+y_{1} z_{1}$

$\frac{d y_{1}}{d t}=-y_{1}-x_{1} z_{1}+\gamma z_{1}$

$\frac{\mathrm{d} z_{1}}{\mathrm{~d} t}=\sigma\left(y_{1}-z_{1}\right)$

$\frac{d x_{2}}{d t}=-x_{2}+y_{2} z_{2}+u_{3}$

$\frac{d y_{2}}{d t}=-y_{2}-x_{2} z_{2}+\gamma z_{2}+u_{4}$

$\frac{d z_{2}}{d t}=\sigma\left(y_{2}-z_{2}\right)$,
Fig. 4 Time series of $x, y, z$ and the output of the controller $u_{2}$ for $\sigma=5.46$ and $\gamma=20$. The initial conditions are $(x(0)$ $y(0), z(0))=(0.01,0.01,0.01)$
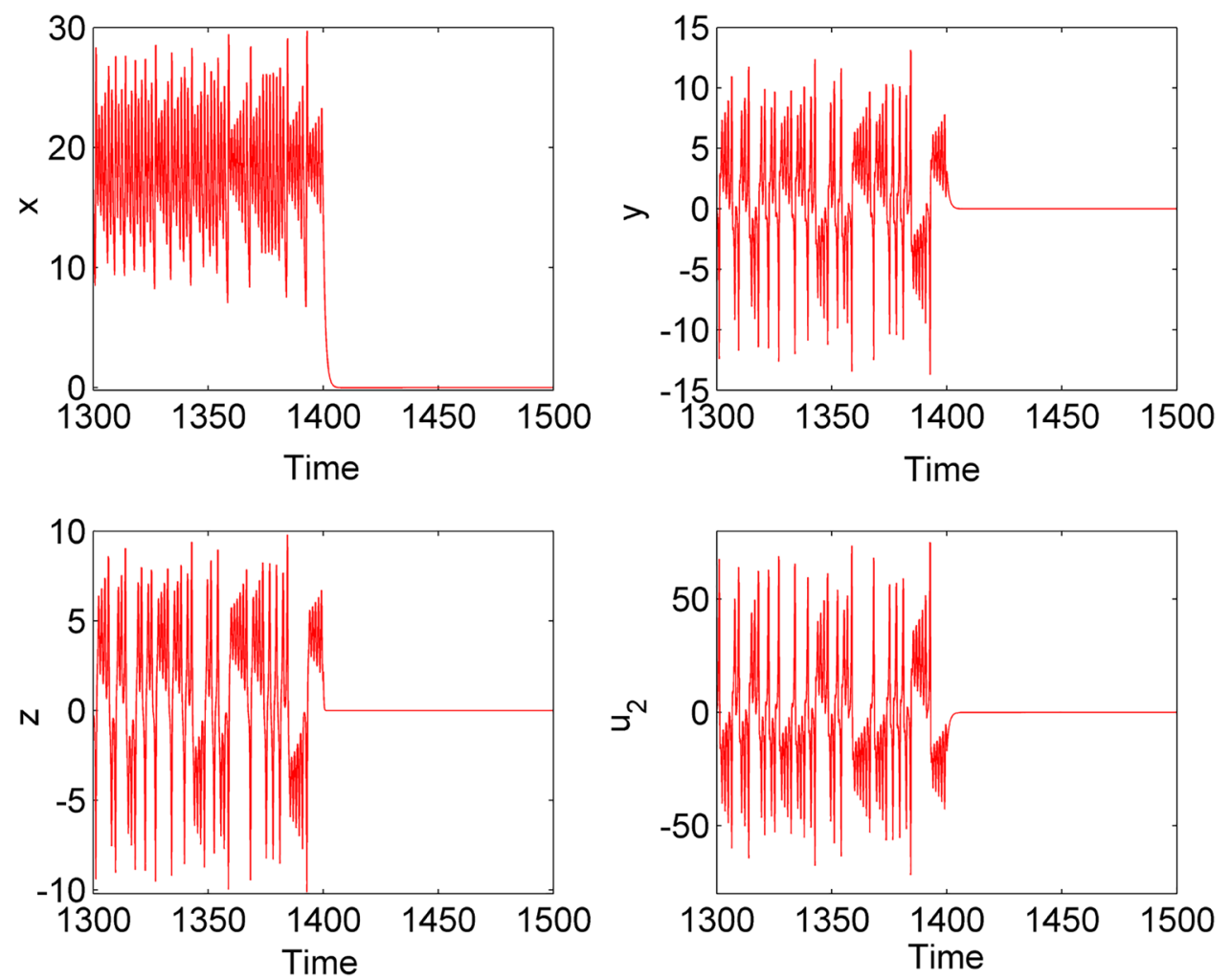
Fig. 5 Time series of synchronization error variables for $\sigma=5.46$ and $\gamma=20$

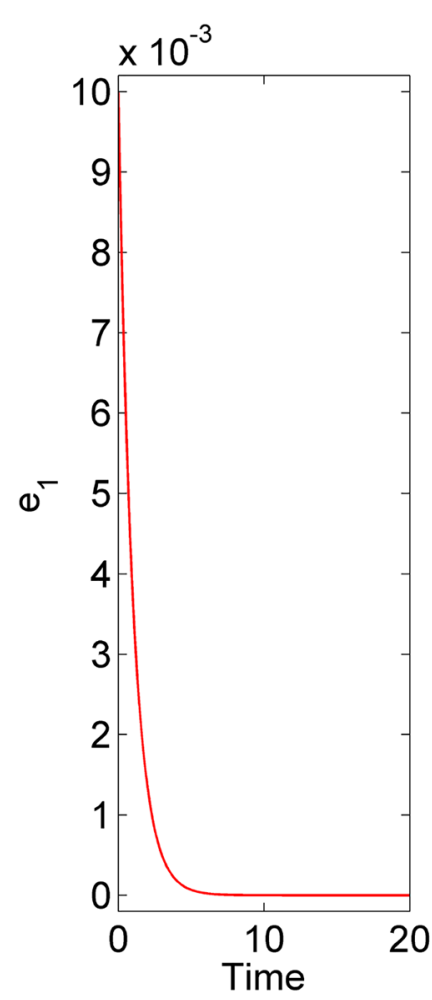

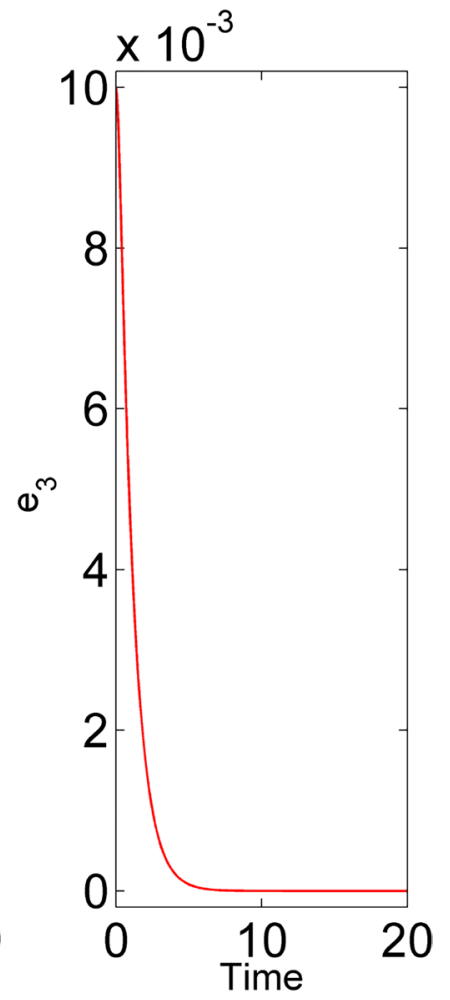

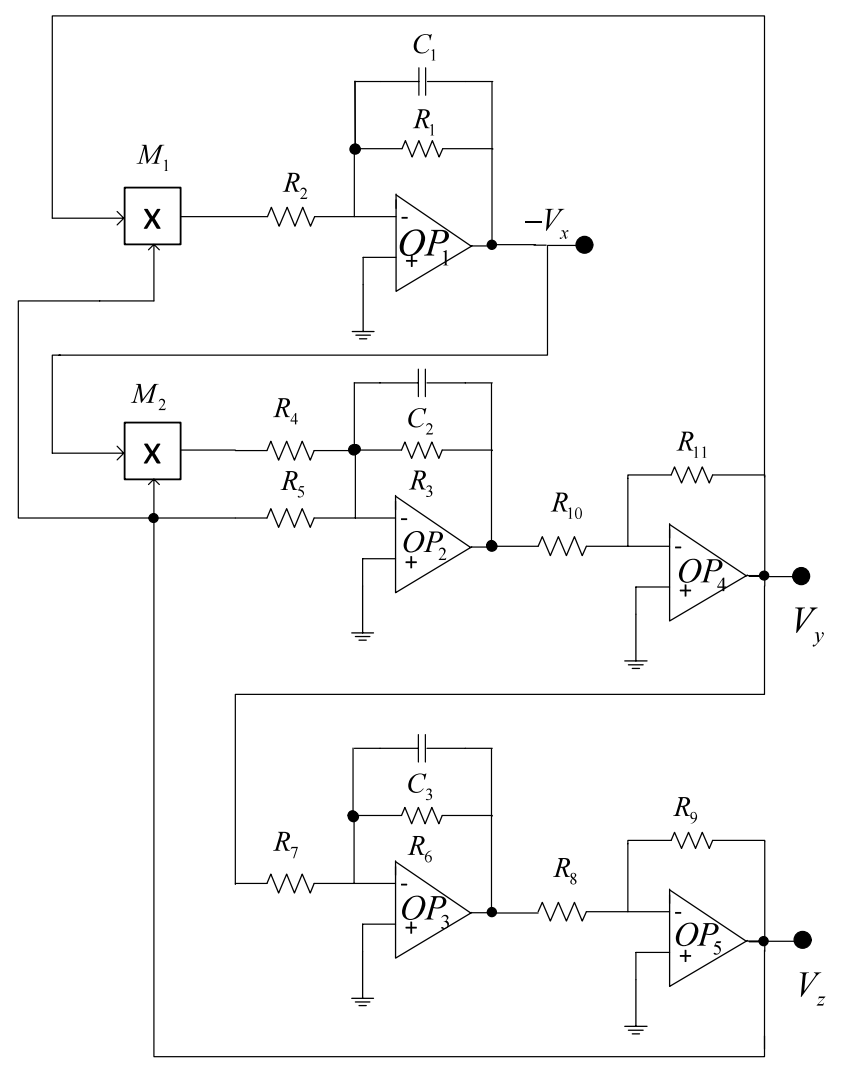

Fig. 6 Circuit diagram of the system (4) describing the PMSM where $u_{3}, u_{4}$ are the controllers to be determined in order to achieve synchronization between the systems (13) and (14). The synchronization errors are defined as $e_{1}=x_{2}-x_{1}, e_{2}=y_{2}-y_{1}, e_{3}=z_{2}-z_{1}$. Its derivatives are given as follows:

$\frac{\mathrm{d} e_{1}}{\mathrm{~d} t}=-e_{1}+z_{2} e_{2}+y_{1} e_{3}+u_{2}$

$\frac{\mathrm{d} e_{2}}{\mathrm{~d} t}=-e_{2}+\gamma e_{3}-z_{2} e_{1}-x_{1} e_{3}+u_{3}$

$\frac{\mathrm{d} e_{3}}{\mathrm{~d} t}=\sigma\left(e_{2}-e_{3}\right)$,

where the controllers are chosen as $u_{3}=-z_{2} e_{2}-y_{1} e_{3}$ and $u_{4}=z_{2} e_{1}+x_{1} e_{3}-\gamma e_{3}$. Substituting the expressions of the controllers $u_{3}, u_{4}$ into the system (15) becomes

$\frac{\mathrm{d} e_{1}}{\mathrm{~d} t}=-e_{1}$

$\frac{\mathrm{d} e_{2}}{\mathrm{~d} t}=-e_{2}$ 


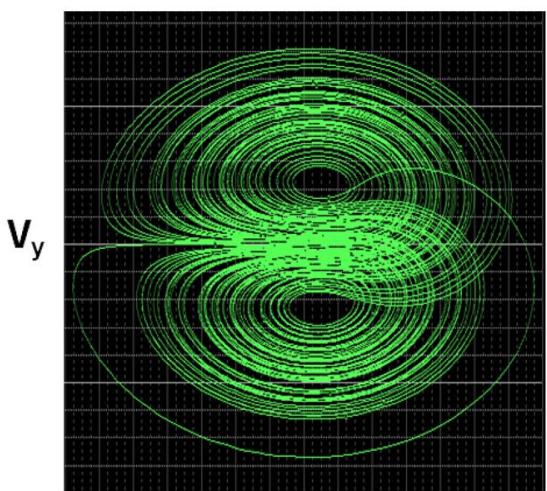

$\mathrm{v}_{\mathrm{x}}$

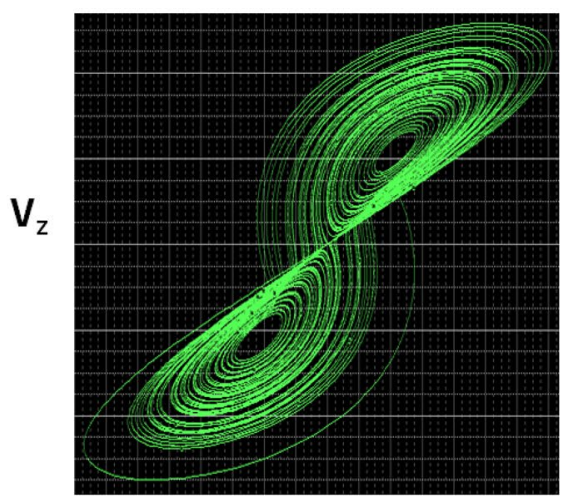

$\mathrm{V}_{\mathrm{y}}$

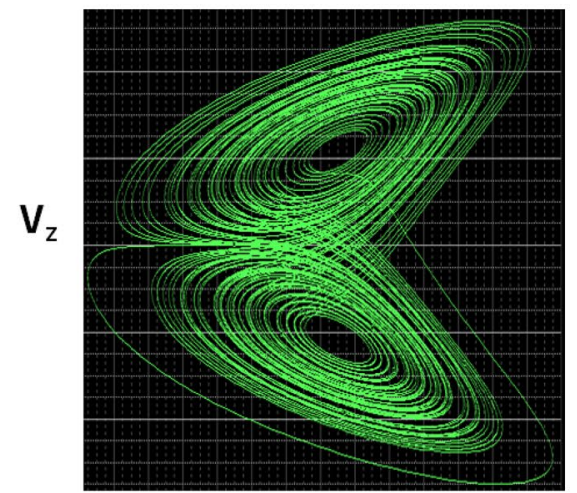

$\mathrm{V}_{\mathrm{x}}$

Fig. 7 (Color online) The phase portraits in planes $\left(V_{x}, V_{y}\right),\left(V_{y}, V_{z}\right)$ and $\left(V_{x}, V_{z}\right)$ of the chaotic attractors observed on the PSpice oscilloscope. Electronic components' values are in the text

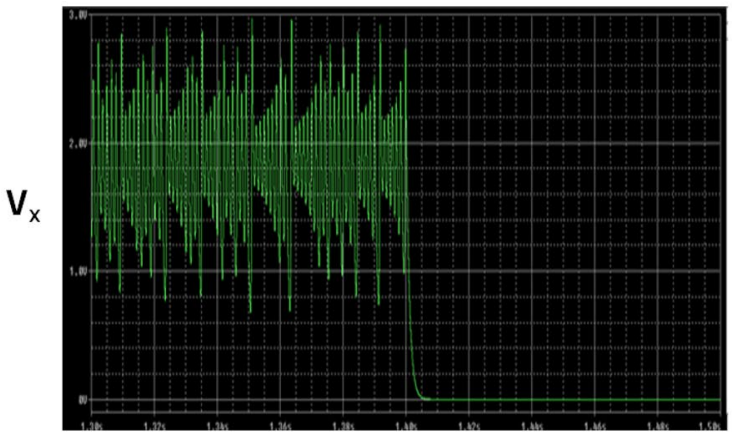

Time

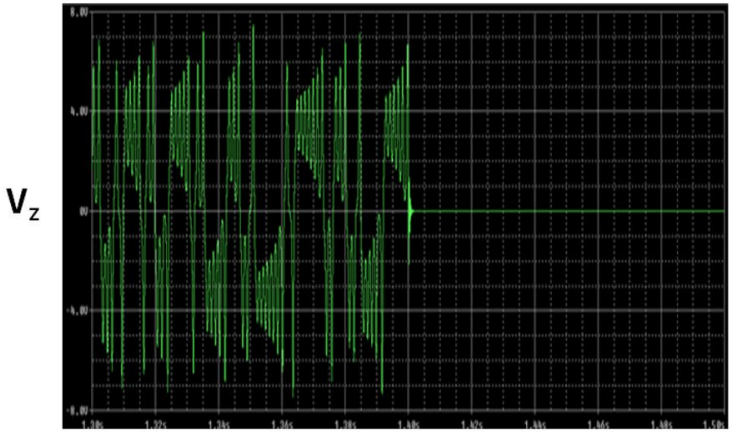

Time

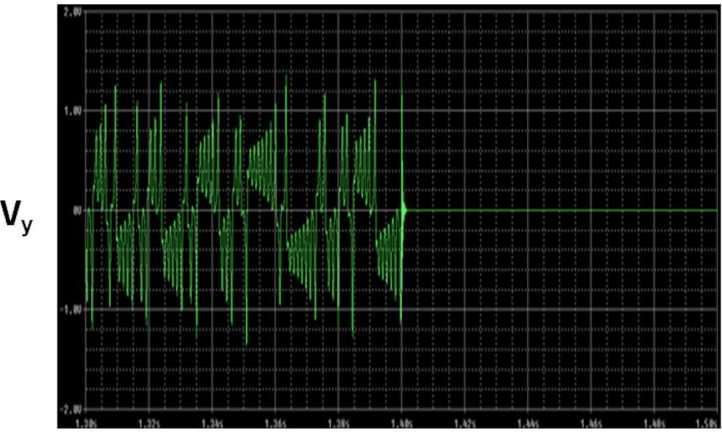

Time

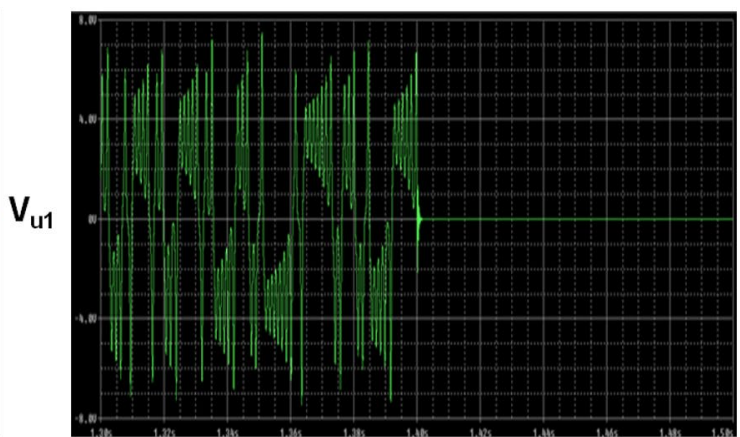

Time

Fig. 8 (Color online) Time series of $V_{x}, V_{y}, V_{z}, V_{u_{1}}$ observed on the PSpice oscilloscope for the capacitors and resistors $C_{1}=C_{2}=C_{3}=10 \mathrm{nF}$ ${ }_{,} R_{1}=R_{3}=100 \mathrm{k} \Omega, R_{2}=R_{4}=10 \mathrm{k} \Omega, R_{5}=5 \mathrm{k} \Omega, R_{6}=R_{7}=18.315 \mathrm{k} \Omega, R_{8}=R_{9}=R_{10}=R_{11}=1 \mathrm{k} \Omega$

$\frac{\mathrm{d} e_{3}}{\mathrm{~d} t}=\sigma\left(e_{2}-e_{3}\right)$

The solutions of Eqs. (16a) and (16b) are $e_{1}(t)=e_{1}(0) e^{-t}$ and $e_{2}(t)=e_{2}(0) e^{-t}$, respectively. That yields $\lim _{t \rightarrow \infty} e_{1}(t)=0$ and $\lim _{t \rightarrow \infty} e_{2}(t)=0$. Thus, the system (16) can be reduced as follows: $\frac{\mathrm{d} e_{3}}{\mathrm{~d} t}=-\sigma e_{3}$.

It is easy to see that $e_{3}(t)=e_{3}(0) e^{-\sigma t}$. Therefore, the following theorem is given:

Theorem 2 The controllers $u_{3}=-z_{2} e_{2}-y_{1} e_{3}$ and $u_{4}=z_{2} e_{1}+x_{1} e_{3}-\gamma e_{3}$ can synchronize the drive system (13) and the response system (14). 


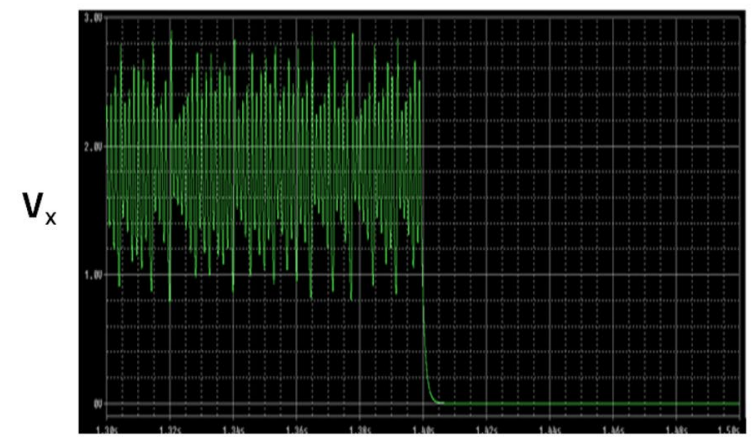

Time

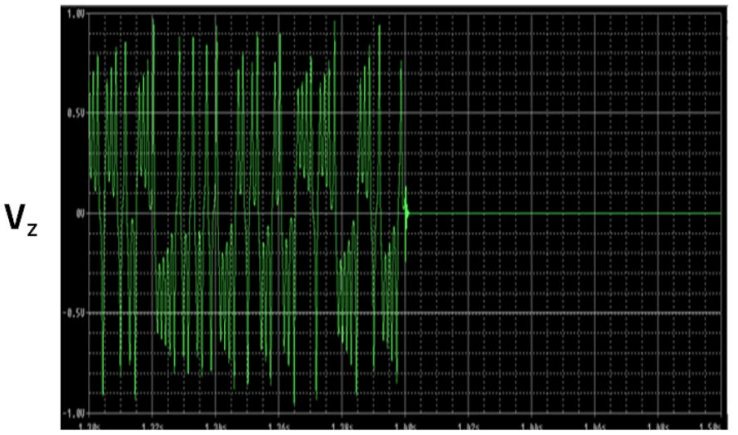

Time

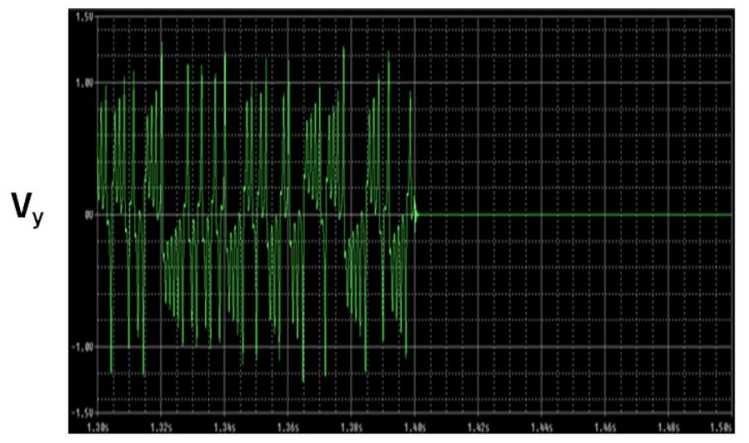

Time

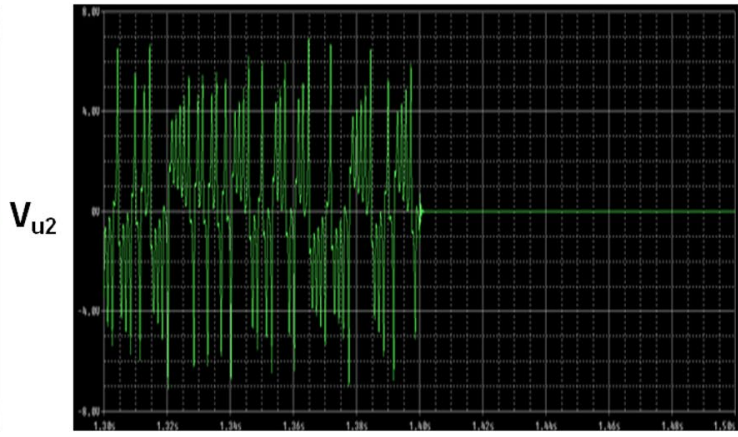

Time

Fig. 9 Time series of $V_{x}, V_{y}, V_{z}, V_{u_{2}}$ observed on the PSpice oscilloscope for the capacitors and resistors $C_{1}=C_{2}=C_{3}=10 \mathrm{nF}$, $R_{1}=R_{3}=100 \mathrm{k} \Omega, R_{2}=R_{4}=10 \mathrm{k} \Omega, R_{5}=5 \mathrm{k} \Omega, R_{6}=R_{7}=18.315 \mathrm{k} \Omega, R_{8}=R_{9}=R_{10}=R_{11}=1 \mathrm{k} \Omega$

Proof The proof is obvious, so leave it out.

For numerical simulations, the initial conditions of the drive system (13) and the response system (14) are $\left(x_{1}(0), y_{1}(0), z_{1}(0)\right)=(0.01,0.01,0.01)$ and $\left(x_{2}(0), y_{2}(0), z_{2}(0)\right)=(0.02,0.02,0.02)$, respectively. The dynamics of the synchronization errors for $\sigma=5.46$ and $\gamma=20$ is depicted in Fig. 5 .

In Fig. 5, the synchronization error variables converge to zero with exponentially asymptotical speed when $t>6.0$.

\section{Circuit implementation}

The existence of chaotic behavior in PMSM and the physical feasibility of the proposed controllers as well as the chaos synchronization of unidirectional coupled PMSMs are tested in this section by designing electronic circuits. The analogue circuitry of the PMSM described by system (4) is shown in Fig. 6.

The electronic circuit of Fig. 6 consists of three capacitors, thirteen resistors, six TL081 operational amplifiers and two analog devices, i.e., AD633 multipliers. Using the following values of parameters: $\omega=1000 \mathrm{rad} \mathrm{s}^{-1}$, $C_{1}=C_{2}=C_{3}=10 \mathrm{nF}, R_{1}=R_{3}=100 \mathrm{k} \Omega, R_{2}=R_{4}=10 \mathrm{k} \Omega$,
$R_{5}=5 \mathrm{k} \Omega$ (for $\left.\gamma=20\right), R_{6}=R_{7}=18.315018 \mathrm{k} \Omega$ (for $\sigma=5.46), R_{8}=R_{9}=R_{10}=R_{11}=1 \mathrm{k} \Omega$, the phase portraits of chaotic behavior found in the PMSM generated from the circuit diagram of Fig. 6 are shown in Fig. 7.

The existence of a double-scroll chaotic attractor found in PMSM can be clearly seen from Fig. 7. By comparing Fig. 7 with Fig. 2, it can be concluded that a good qualitative agreement between the numerical simulations and the PSpice results is obtained. The analogue circuitries of the controlled systems (6) and (10) are deduced from the circuit diagram of system (4) in Fig. 5 (not shown). The time series of the state responses and the output of the controller 1 generated from the circuit diagram of the controlled system (6) are shown in Fig. 8.

In Fig. 8, the matching of PSpice results with the numerical simulation results of Fig. 3 signifies the feasibility of the proposed controller 1 . The time series of the state responses and the output of the controller 2 generated from the circuit diagram of the controlled system (10) are shown in Fig. 9.

In Fig. 9, the matching of PSpice results with the numerical simulation results of Fig. 4 signifies the feasibility of the proposed controller 2 . The analogue circuitry of the chaos synchronization of unidirectional coupled identical PMSMs is depicted in Fig. 10.

\section{SN Applied Sciences}




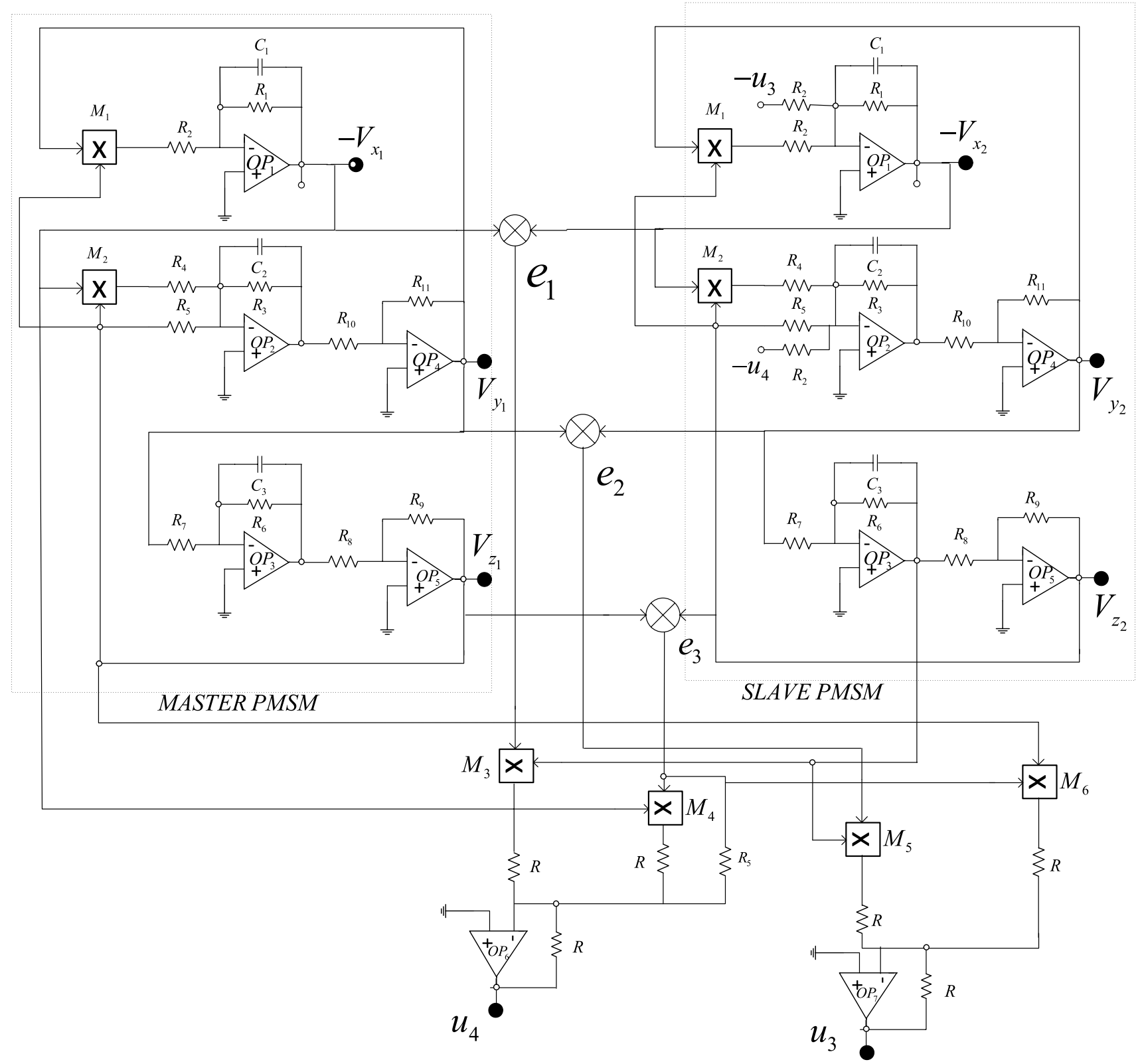

Fig. 10 Circuit diagram of the drive system (13) and the response system (14)

The electronic circuit of Fig. 10 consists of six capacitors, forty-nine resistors, eighteen TL081 operational amplifiers and eight analog devices, i.e., AD633 multipliers. The time series of the synchronization error variables generated from the circuit diagram of Fig. 10 are presented in Fig. 11.

In Fig. 11, the good agreement of PSpice results with the numerical simulation results of Fig. 5 signifies the feasibility of the chaos synchronization of unidirectional coupled identical PMSMs.

\section{Conclusion}

This paper dealt with chaos control via single-state feedback control and synchronization of chaotic permanent magnet synchronous motor. The bifurcation diagram corresponding to two specific parameters of permanent magnet synchronous motor was established and shown to exhibit rich dynamic features. This bifurcation helps in the design of a permanent magnet synchronous motor. Based on the Routh-Hurwitz criteria, the two proposed single simple controllers were designed to suppress the chaotic behavior found in permanent magnet synchronous 


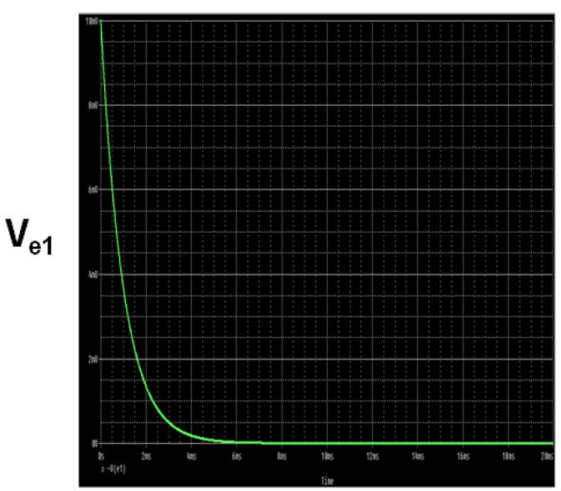

Time

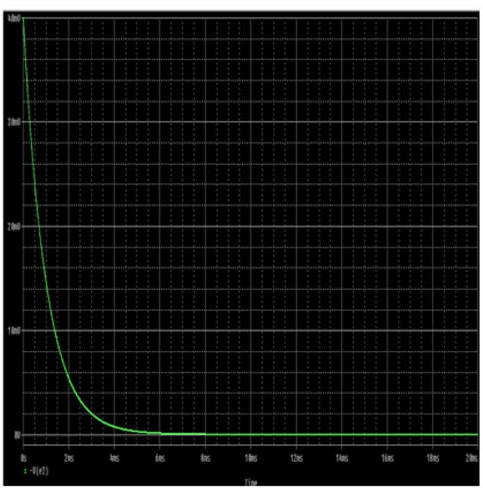

Time

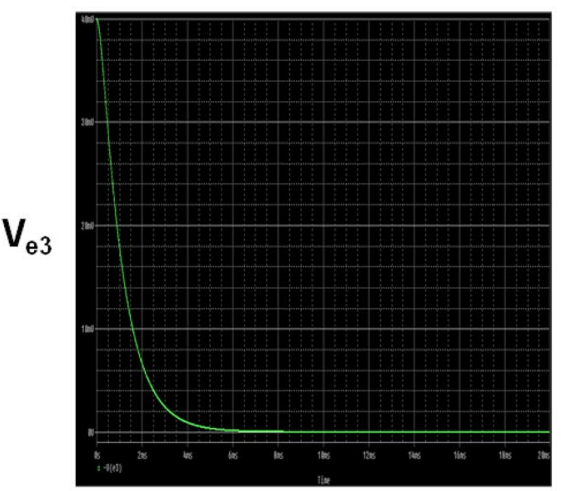

Time

Fig. 11 (Color online) Time series of synchronization error variables $V_{e_{1}}, V_{e_{2}}, V_{e_{3}}$ observed on the PSpice oscilloscope

motor. Among these two simple and single controllers, the simple and single controller 2 could be a preferable choice because of its use of one state variable (i.e., y). Moreover, the synchronization of unidirectional coupled identical chaotic permanent magnet synchronous motors was achieved using simple controllers. Finally, in order to access the physical feasibility of the two proposed simple and single controllers, chaos synchronization and the existence of chaos in permanent magnet synchronous motors were implemented and validated on OrCADPSpice software. The novelty of this paper is to design and implement using analogue circuitry simple, single and effective controllers to suppress chaotic behavior in permanent magnet synchronous motor. In future works, it will be interesting to check if it is possible to design simple and single controllers effective to suppress chaotic behavior in other servo drive systems such as brushless DC motor and synchronous reluctance motor.

Acknowledgements A.C. wishes to thank Prof. John Mucho Ngundam (University of Yaounde I, Cameroon) for his valuable teachings. S.T.K. wishes to thank Dr. Otti D'Huys (Aston University, Birmingham, UK) for careful reading of the manuscript and Prof. Paul Woafo (University of Yaounde I, Cameroon) for his valuable teachings. A.C.C. is grateful to Prof. René Yamapi (University of Douala, Cameroon) and Prof. Paul Woafo (University of Yaounde I, Cameroon) for their valuable teachings and their fruitful advice.

\section{Compliance with ethical standards}

Conflict of interest On behalf of all the authors, the corresponding author declares that there is no conflict of interest in this research article.

\section{References}

1. Ataei M, Kiyoumarsi A, Ghorbani B (2010) Control of chaos in permanent magnet synchronous motor by using optimal Lyapunov exponents placement. Phys Lett A 374:4226-4230

2. Babaei M, Nazarzadeh J, Faiz J (2008) Nonlinear feedback control of chaos in synchronous reluctance motor drive systems. In: IEEE international conference on industrial technology, Chengdu, China, pp 1-5

3. Blasius B, Huppert A, Stone L (1999) Complex dynamics and phase synchronization in spatially extended ecological systems. Nature 399:354-359

4. Cahill DPM, Adkins B (1962) The permanent magnet synchronous motor. Proc Inst Elec Eng A 109:483-491

5. Chen Q, Nan Y, Zheng H, Ren X (2015) Full-order sliding mode control of uncertain chaos in a permanent magnet synchronous motor based on a fuzzy extended state observer. Chin Phys B 24:110504

6. Chen X, Hu J, Peng Z, Yuan C (2017) Bifurcation and chaos analysis of torsional vibration in a PMSM-based driven system considering electromechanically coupled effect. Nonlinear Dyn 88:277-292

7. Choi HH (2012) Adaptive control of a chaotic permanent magnet synchronous motor. Nonlinear Dyn 69:1311-1322

8. Chua LO, Itoh M, Kocarev L, Eckert K (1993) Chaos synchronization in Chua's circuit. J Circuits Syst Comput 3:93-108

9. Cuenot J, Zaim S, Nahid-Mobarakeh B, Pierfederici S, Monmasson E, Meuret R, Meibody-Tabar F (2017) Overall size optimization of a high-speed starter using a quasi-Z-source inverter. IEEE Trans Transp Electr 3:891-900

10. Dong L, Long WS, Hong ZX (2008) Impulsive control in permanent magnet synchronous motor with parameters uncertainties. Chin Phys B 17:1678-1684

11. Hong DK, Wang WH, Lee JY, Woo BC (2018) Design, analysis, and experimental validation of a permanent magnet synchronous motor for articulated robot applications. IEEE Trans Magn 54:1-4

12. Hu J, Liu L, Ma D-W (2014) Robust nonlinear feedback control of a chaotic permanent-magnet synchronous motor with a load torque disturbance. J Korean Phys Soc 65:2132-2139

13. lqbal A, Singh GK (2019) Chaos control of permanent magnet synchronous motor using simple controllers. Trans Inst Meas Control 41:2352-2364 
14. Jian H, Yang Q, Hui L (2016) Adaptive robust nonlinear feedback control of chaos in PMSM system with modeling uncertainty. Appl Math Model 40:8265-8275

15. Kommuri SK, Defoort M, Karimi HR, Veluvolu KC (2016) A robust observer-based sensor fault-tolerant control for PMSM in electric vehicles. IEEE Trans Ind Electron 63:7671-7681

16. Li Z, Zhang B, Tian L, Mao Z, Pong MH (1999) Strange attractors in permanent-magnet synchronous motors. In: IEEE Int Conf Power electronics and drive system (Hong Kong), pp 150-155

17. Li Z, Zhang B, Mao Z (2000) Analysis of the chaotic phenomena in permanent-magnet synchronous motors based on Poincare map. In: Proc. 3rd World Congress intelligent control and automation (Hefei, China), pp 3255-3258

18. Li Z, Park JB, Joo YH, Zhang B, Chen G (2002) Bifurcations and chaos in a permanent magnet synchronous motor. IEEE Trans Circuits Syst-I Fund Thoery Appl 49:383-387

19. Li C, Liao X, Wong K (2004) Chaotic lag synchronization of coupled time-delayed systems and its applications in secure communication. Physica D 194:187-202

20. Liao X, Yu P (2008) Absolute stability of nonlinear control systems. Springer, New York

21. Luo S (2014) Adaptive fuzzy dynamic surface control for the chaotic permanent magnet synchronous motor using nussbaum gain. Chaos 24:1-10

22. Luo S, Wang J, Zhen S, Qian Q (2014) Output feedback adaptive dynamic surface control of permanent magnet synchronous motor with uncertain time delays via RBFNN. Discret Dyn Nat Soc 2014:1-12

23. Maeng G, Han HC (2013) Adaptive sliding mode control of a chaotic nonsmooth-air-gap permanent magnet synchronous motor with uncertainties. Nonlinear Dyn 74:571-580

24. Mao W, Liu G (2019) Development of an adaptive fuzzy sliding mode trajectory control strategy for two-axis PMSM-driven stage application. Int J Fuzzy Syst 21(3):793-808

25. Qi DL, Wang JJ, Zhao GZ (2005) Passive control of permanent magnet synchronous motor chaotic system. J Zhejiang Univ Sci A 6:728-732

26. Qiu H, Tang B, Wang R, Zhang G, Yang C, Cui G (2016) Optimization and calculation of equivalent thermal network method in the temperature field research of permanent magnet servo motor. Recent Adv Electr Electron Eng 9:241-248

27. Qu WD, Bo Z (2009) Controlling chaos in permanent magnet synchronous motor based on finite-time stability theory. Chin Phys B 18:1399-1403
28. Raafat SM, Akmeliawati Rini (2012) Survey on robust control of precision positioning systems. Recent Patents Mech Eng 5:55-68

29. Singh Shweta, Tiwari AN, Singh SN (2018) Sensor-based and sensorless vector control of permanent magnet synchronous motor drives: a comparative study. Recent Adv Electr Electron Eng 11:1-7

30. Singh JP, Roy BK, Kuznetsov NV (2019) Multistability and hidden attractors in the dynamics of permanent magnet synchronous motor. Int J Bifurc Chaos 29:1950056-1950072

31. Su K, Li C (2014) Chaos control of permanent magnet synchronous motors via unidirectional correlation. Optik-Int J Light Electron Opt 125:3693-3696

32. Wang X-J, Rinzel J (1992) Alternating and synchronous rhythms in reciprocally inhibitory model neurons. Neural Comput 4:84-97

33. Wang L, Fan J, Wang Z, Zhan B, Li J (2016) Dynamic analysis and control of a permanent magnet synchronous motor with external perturbation. J Dyn Syst Meas Contr 138:011003-011009

34. Wei D-Q, Zhang B, Luo X-S, Qiu D-Y (2013) Nonlinear dynamics of permanent-magnet synchronous motor with $\mathrm{v} / \mathrm{f}$ control. Commun Theor Phys 59:302-306

35. Wu Y, Li G (2018) Adaptive disturbance compensation finite control set optimal control for PMSM systems based on sliding mode extended state observer. Mech Syst Signal Process 98:402-414

36. Wu X, Tian R, Rui X (2018) Adaptive robust speed control for continuously variable transmission in wind turbine under grid faults. J Renew Sustain Energy 10:063302

37. Yu J, Gao J, Ma Y et al (2010) Robust adaptive fuzzy control of chaos in permanent magnet synchronous motor. Discret Dyn Nat Soc Article ID 269283, pp 1-10

38. Yu J, Chen B, Yu H, Gao J (2011) Adaptive fuzzy tracking control for the chaotic permanent magnet synchronous motor drive system via backstepping. Nonlin Anal Real World Appl 12:671-681

39. Yuanzi D, Soon LK, Xiuli G (2001) Digital control of a permanent magnet synchronous motor drive without mechanical sensors. Electr Power Compon Syst 29:459-477

Publisher's Note Springer Nature remains neutral with regard to jurisdictional claims in published maps and institutional affiliations. 